\title{
Arthroscopic synovectomy and radiosynoviorthesis: a treatment option for recurrent arthritis symptoms in patients with pachydermoperiostosis
}

\author{
S. Warwas ${ }^{1}$, C. Specker ${ }^{2}$, M. Jäger ${ }^{1}$, S. Landgraeber ${ }^{1}$ \\ ${ }^{1}$ Department of Orthopaedics, University of Duisburg-Essen; \\ ${ }^{2}$ Department of Rheumatology, Kliniken Essen-Sued, Essen, Germany
}

\section{SUMMARY}

Pachydermoperiostosis as the primary form of hypertrophic osteoarthropathy is a rare hereditary disorder with a number of characteristic findings, e.g. periosteal hypertrophy, digital clubbing and pachydermia. Further typical symptoms include arthritic manifestations in all major joints. Literature review showed that therapeutical approaches usually focus on medical therapy alleviating chronic polyarthritis by controlling inflammation. In the case presented in this report anti-inflammatory therapy appeared to have been insufficient since knee arthritis persisted. Arthroscopic synovectomy and radiosynoviorthesis were performed consecutively. Postoperative and follow-up examinations indicate that this combined therapy reduces pain, increases range of motion and improves overall mobility, and is thus of lasting benefit to the patient.

Key words: Pachydermoperiostosis, Arthritis, Osteoarthropathy, Arthroscopy.

Reumatismo, 2013; 65 (2): 82-85

\section{INTRODUCTION}

$\mathrm{P}$ achydermoperiostosis (PDP) is the primary form of hypertrophic osteoarthropathy (HOA). It is a rare hereditary disorder first described in 1868 by Friedreich (1) and is also referred to as TouraineSolente-Golé syndrome. The precise incidence is unknown. PDP is assumed to be inherited in an autosomal dominant pattern and is to be found predominantly in men. However, autosomal recessive forms have also been found (2). Recent studies revealed mutations in the 15-hydroxyprostaglandin dehydrogenase gene and the prostaglandin transporter SLCO2A1 to be possible causes of PDP $(3,4)$. Phenotypic and radiographic characteristics include periostosis, pachydermia and finger clubbing (5). Further typical clinical findings are polyarthritis, cutis verticis gyrata, hyperhidrosis, seborrhea and eyelid thickening and ptosis. Department of Orthopaedics Hufelandstrasse, 55 - 45147 Essen, Germany E-mail: sebastian.warwas@uk-essen.de
In this article a PDP-case with recurrent severe arthritis symptoms despite long-term treatment with anti-inflammatory drugs (NSAID) and steroids is reported.

\section{CASE REPORT}

A 39-year-old man of Aramaic-Iraqi origin with recurrent painful swelling of all major joints, especially the left knee, is presented. This patient had been diagnosed with PDP just one year before. Anamnesis revealed that first symptoms, e.g. widening of the fingers, already started at the age of 17 . According to the patient, two brothers were affected by similar symptoms whereas the consanguineous parents were not.

Physical examination showed the full spectrum of PDP-characteristic findings with finger clubbing, bilateral ptosis and furrowed facial skin. Additionally, severe arthritis with swelling of the knees, ankles and finger joints together with edema of the lower legs were found. Radiographic examination revealed typical periosteal proliferation and secondary arthrosis of all 
major joints. Although C-reactive protein was elevated to $6.7 \mathrm{mg} / \mathrm{dL}$ (normal value: $0.5 \mathrm{mg} / \mathrm{dL}$ ) no local or systemic infection could be identified.

Before presenting at our clinic the patient had been treated with bisphosphonates, NSAID and steroids. An operation had been performed on both eyelids to reduce mechanical sight impairment. Since months severe arthritic symptoms regularly recurred in the left knee with painful swelling, reduced range of motion (ROM) with flexion limited to 90 degrees and impaired overall mobility. Magnetic resonance image of the left knee revealed massive intraarticular effusion (Fig. 1). In the right knee the symptoms were considerably milder.

Since the left knee was worst affected and seemed not to have responded to the treatment given so far, an arthroscopy associated with synovectomy was performed. Intra-operative findings showed a massive increase in volume of the synovial membrane in the context of chronic synovitis and lipomatosis. Additionally, this tissue appeared highly vascularized (Fig. 2). The bone, cartilage and ligaments were in good shape but partially covered by hypertrophic soft synovial tissue. Synovectomy was complicated by recurrent bleeding due to hypervascularization. Using a tourniquet cuff on the upper leg and performing synovectomy rather by radiofrequency ablation than by mechanical shaving helped managing this difficulty sufficiently. In some areas of the joint synovectomy was limited since proliferated soft tissue and underlying bone and cartilage appeared to have grown together. However, after a time consuming procedure, the hypertrophic synovial membrane was almost completely removed. Tissue samples were collected for histopathological analysis. The microscopic assessment corresponded to the intraoperative findings such as chronic synovitis, infiltrated with lymphocytes and plasma-cells, severe lipomatosis and increased vascularization.

The postoperative period was initially characterized by recurrent hemarthrosis of the knee which had to be punctured twice. However, the joint was mobilized continuously starting one day after surgery

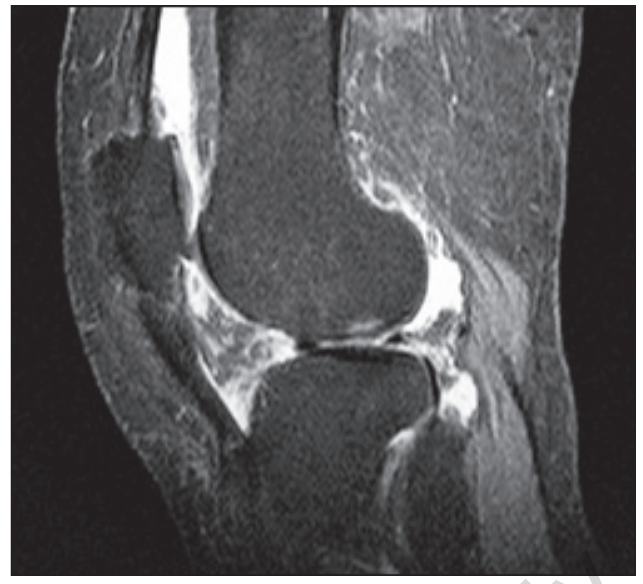

Figure 1 - Magnetic resonance image of the left knee showing distinct intra-articular effusion.

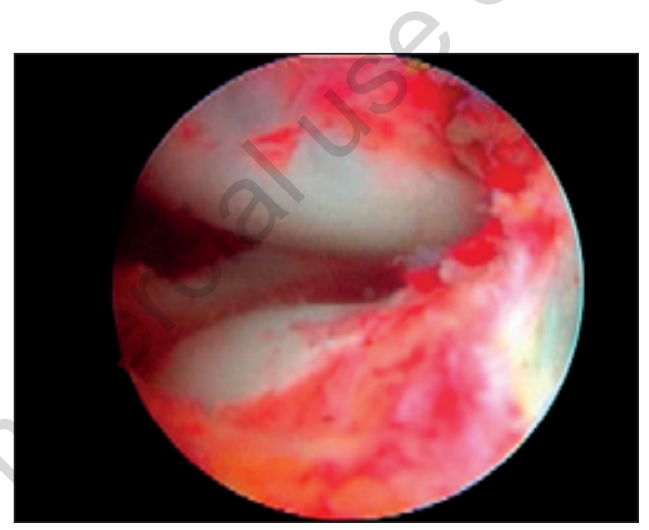

Figure 2 - Macroscopic view revealing highly vascularized fatty synovial proliferation.

to prevent a decrease in ROM. From the fourth day on a significant reduction of all symptoms followed. At discharge, the patient showed an improved ROM allowing maximum flexion of up to 120 degrees. Improved mobility and distinct pain relief were also observed. Nevertheless, since complete synovectomy could be hardly achieved by arthroscopy, radiosynoviorthesis of the left knee was additionally performed six weeks after surgery.

The case was followed-up one year after the operation. All symptoms in the left knee remained significantly improved. The pain was still markedly decreased. Joint effusions appeared regressive and the circumference of the joint was measurably reduced. The patient was considerably 
more mobile than before arthroscopy, and flexion of the left knee was still possible up to 120 degrees. According to the patient, the success of the therapy was particularly due to the arthroscopic synovectomy, but radiosynoviorthesis had brought further relief. The patient assessed the treatment of his knee as generally very good and would be prepared to undergo the same therapy again at any time if necessary.

\section{DISCUSSION}

PDP as the primary form of HOA is a rare hereditary disorder with a couple of characteristic findings, e.g. periosteal hypertrophy, digital clubbing and pachydermia (5). Further typical symptoms include arthritis manifestations in all major joints (6). HOA-associated arthritis is considered to be reactive.

This is due to cytokine-mediated tissue remodelling, endothelial cell activation and increased vascular stimulation leading to chronic hypertrophic synovitis (7). Since arthritis may have its cause in a variety of underlying diseases, differential diagnoses (e.g. rheumatic diseases) have to be considered or excluded $(8,9)$. Therapeutical approaches usually aim at alleviating chronic polyarthritis and reducing hypertrophy of the synovial membrane by controlling inflammation. Drug therapy includes the use of NSAIDs and steroids. Furthermore, bisphosphonates may improve symptoms (10). Arthroscopic treatment of PDP including extensive synovectomy was described only once in literature and was proved to be a useful measure, but no details are given about the arthroscopy, as the main interest of that report is not focused on this topic (11).

In this case anti-inflammatory therapy appeared not to have sufficiently relieved all symptoms since signs of arthritis persisted in the left knee. Our postoperative examinations showed an immediate and continuous decrease in arthritis-associated symptoms with pain relief, reduced knee circumference plus an increase in ROM and mobility. The follow-up examination one year later showed that the improvement was not short-lived.

However, since the proliferated synovial tissue was highly vascularized, arthroscopic treatment harbored an increased risk of bleeding complications, so the use of a tourniquet cuff and radiofrequencies are meaningful. Postoperatively, significant hemarthrosis persisted and the knee had to be punctured twice. This circumstance and the underlying chronic inflammatory disease also increase the risk of disturbed wound healing and local infection. The effect of radiosynoviorthesis, which was carried out six weeks postoperatively, also has to be considered when evaluating the results of the follow-up examination. Since beneficial effects were registered directly after arthroscopy and continued improved after radiosynoviorthesis, an increased overall benefit by combining both procedures can be confirmed (12). However, since radiosynoviorthesis bears the risk of bone necrosis, cartilage integrity always has to be ensured before. In summary, arthroscopic synovectomy appears to be an effective procedure for management of recurrent arthritis symptoms in PDP-patients. The benefit of this treatment might even be enhanced by subsequent radiosynoviorthesis. To our knowledge it is the first report about an arthroscopic treatment of PDP in combination with radiosynoviorthesis.

\section{REFERENCES}

1. Friedreich N. Hyperostose des gesamten Skelettes. Virchows Arch J Path Anat. 1868; 43: 83-7.

2. Castori M, Sinibaldi L, Mingarelli R, et al. Pachydermoperiostosis: an update. Clin Genet. 2005; 68: 477-86.

3. Uppal S, Diggle CP, Carr IM, et al. Mutations in 15-hydroxyprostaglandin dehydrogenase cause primary hypertrophic osteoarthropathy. Nat Genet. 2008; 40: 789-93.

4. Zhang Z, Xia W, He J, et al. Exome sequencing identifies SLCO2A1 mutations as a cause of primary hypertrophic osteoarthropathy. Am J Hum Genet. 2012; 13: 125-32.

5. Jajic Z, Jajic I, Nemcic T. Primary hypertrophic osteoarthropathy: clinical, radiologic, and scintigraphic characteristics. Arch Med Res. 2001; 32: 136-42. 
6. Matsumoto T, Tsurumoto T, Shindo H. A case of pachydermoperiostosis associated with arthritis. Mod Rheumatol. 2003; 13: 371-3.

7. Narayanan S, Mohamed Gani VM, Sundararaju V. Primary hypertrophic osteoarthropathy with hypertrophic gastropathy. J Clin Rheumatol. 2010;16: 190-2.

8. Diamond S, Momeni M. Primary hypertrophic osteoarthropathy in a patient with rheumatoid arthritis. J Clin Rheumatol. 2007; 13: 242-3.

9. Guyot-Drouot MH, Solau-Gervais E, Cortet B, et al. Rheumatologic manifestations of pachydermoperiostosis and preliminary ex- perience with bisphosphonates. J Rheumatol. 2000; 27: 2418-23.

10. Bhansali A, Singh R, Sriraam M, Bhadada S. Pachydermoperiostitis and bisphosphonates. J Assoc Physicians India. 2006; 54: 340.

11. Jojima H, Kinoshita K, Naito M. A case of pachydermoperiostosis treated by oral administration of a bisphosphonate and arthroscopic synovectomy. Mod Rheumatol. 2007; 17: 330-2.

12. Kerschbaumer F, Kandziora F, Herresthal J, et al. Combined arthroscopic and radiation synovectomy in rheumatoid arthritis. Orthopade. 1998; 27: 188-96. 